\title{
Vertebrobasilar insufficiency and gamma-glutamyltransferase: An emerging relationship demonstrating inflammation and atherosclerosis
}

\author{
Ulu MS ${ }^{1}$, Beker-Acay $\mathrm{M}^{2}$, Ulu $\mathrm{S}^{3}$, Unlu $\mathrm{E}^{2}$, Bucak $\mathrm{A}^{3}$, Yaman $\mathrm{G}^{1}$, Altug $\mathrm{A}^{1}$, Tekin $\mathrm{MS}^{3}$, Oruc $\mathrm{S}^{4}$ \\ Department of Internal Medicine, Afyon Kocatepe University, Faculty of Medicine, Afyonkarahisar, \\ Turkey.mehtapacay@gmail.com
}

\begin{abstract}
OBJECTIVES: Vertebrobasilar insufficiency (VBI) is a pathology arising from the reduction in flow rate of vertebral arteries and mainly caused by inflammation and atherosclerosis. Gamma-glutamyltransferase (GGT) is a marker which has been recently recognized as a marker of inflammation and atherosclerosis. We aimed to investigate the relationship between GGT levels and VBI for the first time.

METHODS: In this cross-sectional study, of 3100 subjects who had vertebrobasilar doppler ultrasonography (VBU) were evaluated and 1042 of them who met the inclusion criterias were included. VBU reports, GGT levels, blood chemistry, lipid profile were received from patients' files. Patients were evaluated according to VBU measurements and divided into two groups, VBI and non-VBI.

RESULTS: Mean vertebral arterial blood flow volume values were $149.99 \pm 32.93 \mathrm{~mL} / \mathrm{m}$ in VBI group and 286.88 $\pm 70.98 \mathrm{~mL} / \mathrm{m}$ in non-VBI group. Mean GGT and CRP values were significantly higher in the VBI group than in the non-VBY group $(p<0.001)(p<0.001)$, respectively. Vertebral artery blood flow volume was negatively correlated with GGT $(r:-0.208, p<0.001)$ and CRP $(r:-0.119, p<0.001)$.

CONCLUSION: We demonstrated a significant correlation between serum GGT levels and VBI. In addition, higher GGT level was an independent risk factor for the presence of vertebrobasilar system inflammation and atherosclerosis (Tab. 2, Fig. 3, Ref. 27). Text in PDF www.elis.sk.

KEY WORDS: vertebrobasilar insufficiency, inflammation, atherosclerosis, gamma-glutamyltransferase.
\end{abstract}

\section{Introduction}

Plasma gamma-glutamyltransferase (GGT) is a marker of hepatobiliary disease and alcohol consumption. Recently it has been recognized that, serum GGT activity represents a true marker of inflammation and atherosclerosis and has prognostic importance. GGT levels were found to be associated with atherosclerotic risk factors, hypertension, stroke, metabolic syndrome and type 2 diabetes mellitus (DM) (1-4). One U/L higher GGT (on a log scale) was associated with a $54 \%$ increased risk of stroke and a $20 \%$ increased risk of coronary heart disease.

The posterior circulation, which supplies the brainstem and the cerebellum, is supplied by the vertebrobasilar arteries. One fifth of strokes occur in the posterior circulation. Of these, $20 \%$ to $25 \%$ are believed to be due to vertebrobasilar insufficiency (VBI). VBI

${ }^{1}$ Department of Internal Medicine, Afyon Kocatepe University, Faculty of Medicine, Afyonkarahisar, Turkey, ${ }^{2}$ Department of Radiology, Afyon Kocatepe University, Faculty of Medicine, Afyonkarahisar, Turkey, ${ }^{3}$ Department of Otorhinolaryngology, Afyon Kocatepe University, Faculty of Medicine, Afyonkarahisar, Turkey, and ${ }^{4}$ Department of Neurology, Afyon Kocatepe University, Faculty of Medicine, Afyonkarahisar, Turkey

Address for correspondence: M. Beker-Acay, MD, Department of Radiology, Afyon Kocatepe University, Faculty of Medicine, 03200, Afyonkarahisar, Turkey.

Phone: +90505.8409464, Fax: +90. 272.2463300 is a pathology arising from the reduction in flow rate of these arteries or their branches, caused mainly by inflammation and atherosclerosis and presents a complex varied clinical picture (5-9). Although GGT which is a good surrogate marker of atherosclerosis, has been examined in stroke and coronary artery disease; the relation between GGT and VBI has not been evaluated to date $(10,11)$. We aimed to investigate the relationship between GGT levels and VBI for the first time.

\section{Materials and methods}

This cross-sectional study was performed between January 2010 and November 2014 in Afyon Kocatepe University School of Medicine in Internal Medicine, Radiology, and Otorhinolaryngology Departments. A total number of 3100 subjects who had vertebrobasilar doppler ultrasonography were evaluated. Of 3100 subjects, 1042 who met the inclusion criteria were included in the study. Patients who had any disease affecting GGT levels or any systemic disease such as, diabetes mellitus, hyperlipidemia, hypertension, chronic liver disease, alcohol consumption, malignancy, hepatosteatosis, infection, acute or chronic renal failure, coronary artery disease, any inflammatory disease, cerebrovascular disease, smoking history and patients with poor ultrasonographic recording quality and those who did not agree to be included in the study were excluded from the study. 
Patients were questioned for age, sex, smoking, family history, heart disease, any risk factors, dietary compliance and drug use. Vertebrobasilar doppler ultrasonography reports, GGT levels, blood chemistry, lipid profile of the patients were recorded. Patients were evaluated according to vertebrobasilar doppler ultrasonography measurements and divided into two groups as VBI and nonVBI. In total sonographic measurements, subjects with vertebral artery (VA) flow volume less than $200 \mathrm{~mL} / \mathrm{m}$ were designated as VBI group, and subjects with VA flow volume more than 200 $\mathrm{mL} / \mathrm{m}$ were designated as non-VBI group $(12,13)$.

\section{Biochemical and haematological analyses}

Plasma triglyceride, total cholesterol, LDL, HDL concentrations, and fasting glucose were measured using an automated chemistry analyzer (Aeroset; Abbott, Holliston, MN, USA) with commercial kits (Abbott). C-reactive protein (CRP) was measured using an autoanalyzer (Aeroset; Abbott) with a spectrophotometric commercial kit (Scil Diagnostics GmbH, Viernheim, Germany). Serum GGT activities were measured with the enzymatic calorimetric test (Roche/Hitachi analyzer, Mannheim, Germany) and the normal range of GGT activity was identified as 7-49 U/l.

\section{Doppler sonography examinations}

All Doppler sonographic measurements were performed using a Toshiba Aplio system (Tokyo, Japan) and examinations were performed by the same experienced radiologists, who were unaware of clinical data. Doppler sonography examinations were performed in a dimly lighted room with a comfortable temperature $\left(22-24{ }^{\circ} \mathrm{C}\right)$ after an adaptation period for at least 15 min rest in supine position. The vertebral arteries (VA) of both sides were explored with a 7.5-Mhz linear array transducer. All of the patients' bilateral VAs were examined in supine position with the head slightly turned to the opposite side. Flow volume measurements were generally taken in the C4 and C5 intertransverse segment of the VA. Net vertebral artery flow volume was detected by calculating the sum of the right and left vertebral artery flow volumes. The radiologic criterion for VBI was accepted as lower than $200 \mathrm{~mL} / \mathrm{min}$ flow volume of VAs $(12,13)$. All measurements were documented by black-and-white video printer. The complete examination took about 15 min for each case.

\section{Statistical methods}

Continuous variables were presented as mean \pm SD and categorical variables were expressed as percentage. KolmogorovSmirnov test was used to evaluate the distribution of variables. Student's t-test (independent sample t-test) was used for continu-

Tab. 1. Demographic data of the groups.

\begin{tabular}{|c|c|c|c|c|}
\hline Variables & & $\begin{array}{l}\text { VBI group } \\
(n=400)\end{array}$ & $\begin{array}{c}\text { Non-VBI group } \\
(n=642)\end{array}$ & $\mathrm{p}$ \\
\hline Age (year) & & $54.32 \pm 17.85$ & $49.42 \pm 17.27$ & 0.001 \\
\hline \multirow{2}{*}{ Gender * } & Male & $179(\% 44.8)$ & 252(\%39.3) & \multirow{2}{*}{0.080} \\
\hline & Female & $221(\% 55.2)$ & $390(\% 60.7)$ & \\
\hline
\end{tabular}

All parameters are expressed as mean \pm standard deviation unless otherwise stated * Data are expressed as number (\%)
Tab. 2. Laboratory data of the groups.

\begin{tabular}{lccc}
\hline Variables & $\begin{array}{c}\text { VBI group } \\
(\mathrm{n}=400)\end{array}$ & $\begin{array}{c}\text { Non-VBI group } \\
(\mathrm{n}=642)\end{array}$ & $\mathrm{p}$ \\
\hline $\begin{array}{l}\text { Vertebral artery blood } \\
\text { flow volume }\end{array}$ & $149.99 \pm 32.93$ & $286.88 \pm 70.98$ & $<0.001$ \\
\hline GGT $(\mathrm{U} / \mathrm{l})$ & $51.27 \pm 33.31$ & $34.60 \pm 23.23$ & $<0.001$ \\
\hline $\mathrm{CRP}(\mathrm{mg} \backslash \mathrm{dl})$ & $1.63 \pm 2.34$ & $1.05 \pm 2.17$ & $<0.001$ \\
\hline Hb(mgldl) & $13.61 \pm 1.89$ & $13.83 \pm 4.71$ & 0.307 \\
\hline Htc (mgldl) & $40.72 \pm 5.09$ & $42.18 \pm 24.40$ & 0.163 \\
\hline WBC & $2778.96 \pm 3829.56$ & $3116.23 \pm 3951.70$ & 0.198 \\
\hline Total cholesterol (mgldl) & $170.16 \pm 31.10$ & $173.00 \pm 37.19$ & 0.375 \\
\hline Triglycerides (mgldl) & $120.46 \pm 76.11$ & $114.43 \pm 75.36$ & 0.403 \\
\hline LDL (mgldl) & $104.58 \pm 26.26$ & $105.39 \pm 29.048$ & 0.756 \\
\hline HDL (mgldl) & $45.80 \pm 13.31$ & $47.54 \pm 14.25$ & 0.186 \\
\hline
\end{tabular}

All parameters are expressed as mean \pm standard deviation unless otherwise stated GGT - Gamma-glutamyltransferase, CRP - C-reactive protein, Hb - Hemoglobin, Htc - hematocrit, WBC - White blood cell, LDL - Low-density lipoprotein cholesterol, HDL - high-density lipoprotein cholesterol

ous variables with normal distribution and Mann-Whitney U test was used for continuous variables without normal distribution. Chi-square test was used for categorical variables. The parameters were investigated using Spearman/Pearson correlation where appropriate. Valeu p $<0.05$ was accepted as significant level. For statistical calculations, SPSS statistical software (SPSS for Windows, version 17.0. inc. Chicago, IL, USA) was used.

\section{Results}

Of 1042 patients, 400 were with VBI (38.3\%). Mean age in the VBI and non-VBI groups was $54.32 \pm 17.85$ years and 49.42 \pm 17.27 years, respectively. Male/Female ratio of patients with VBI was 179/221, while it was 252/390 inthe non-VBI group. Demographic data of the two groups are demostrated in Table 1.

Mean vertebral artery blood flow volume values were 149.99 $\pm 32.93 \mathrm{~mL} / \mathrm{m}$ in VBI group and $286.88 \pm 70.98 \mathrm{~mL} / \mathrm{m}$ in nonVBI group. Laboratory data of the groups are outlined in Table 2.

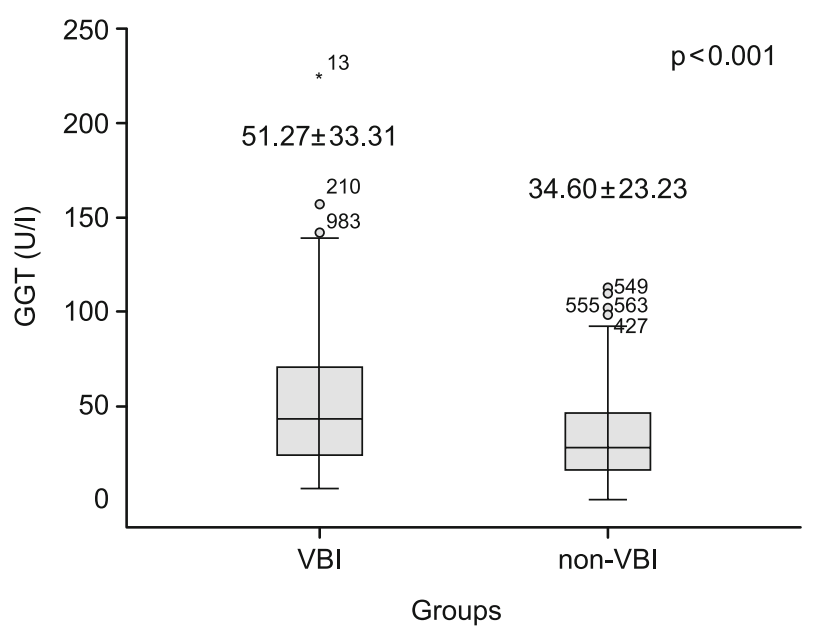

Fig. 1. Mean gamma-glutamyltransferase (GGT) values of the groups. VBI - vertebrobasilar insufficiency. 
448-452

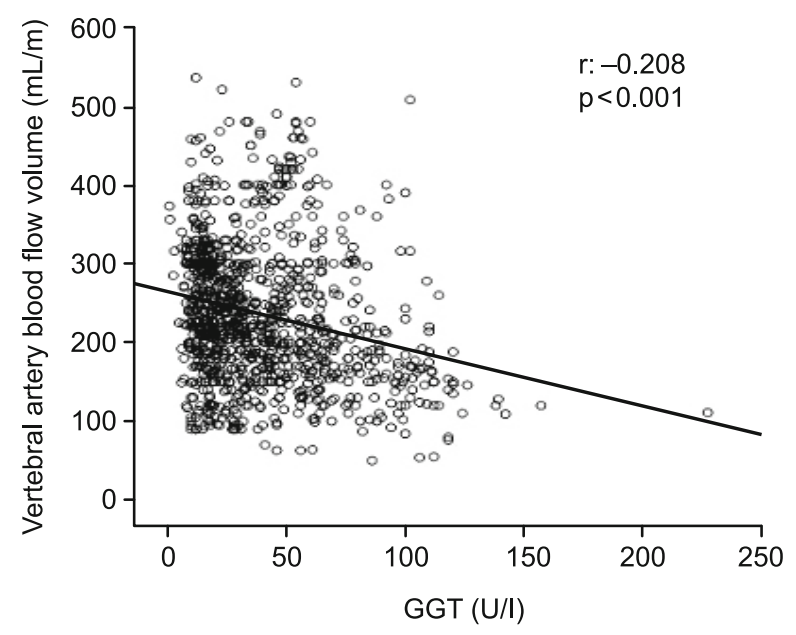

Fig. 2.The relationship between plasma gamma-glutamyltransferase (GGT) and vertebral artery blood flow volume.

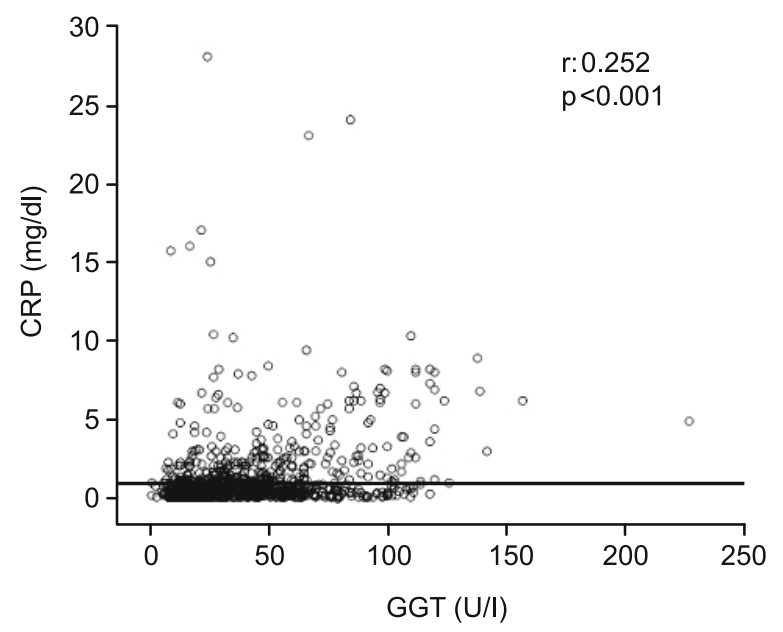

Fig. 3.The relationship between plasma gamma-glutamyltransferase and $\mathrm{C}$-reactive protein levels.

Mean GGT values were $51.27 \pm 33.31 \mathrm{U} / \mathrm{l}$ in VBI group and $34.60 \pm 23.23 \mathrm{U} / \mathrm{l}$ in the non-VBY group. GGT values of the VBI group were significantly higher than in the non-VBY group $(\mathrm{p}<$ 0.001) (Fig. 1). Mean CRP values were $1.63 \pm 2.34 \mathrm{mg} / \mathrm{dl}$ in the VBI group and $1.05 \pm 2.17 \mathrm{mg} / \mathrm{dl}$ in the non-VBY group ( $<0.001$ ).

Vertebral artery blood flow volume was negatively correlated with GGT (r: -0.208, p < 0.001) and CRP (r: -0.119, p < 0.001) (Fig. 2). There was a positive correlation between GGT and CRP levels (r: 0.252, p < 0.001) (Fig. 3). Besides GGT was positively correlated with triglyceride, total cholesterol, LDL and HDL cholesterol.

\section{Discussion}

This is the first study investigating the relationship between VBI, inflammation and atherosclerosis via GGT levels. And our results showed that GGT levels were independently associated with VBI and reduction in flow rate of vertebrobasilar system.

Although the evaluation of serum GGT is a well-established diagnostic test for hepatobiliary disease, recently it was found as a reliable marker of atherosclerosis and shown in many inflammatory and atherosclerotic conditions. Higher serum GGT activity has been reported in atherosclerotic plaques and foam cells $(14,15)$.

Epidemiologic studies have reported that GGT is significantly associated with cardiovascular mortality and stroke $(16,17)$. Dogan et al showed a relationship between higher GGT concentrations and coronary atherosclerosis (18). Shankar et al found a positive association between GGT level and peripheral arterial atherosclerosis (19). Moreover, serum GGT activity was associated with carotid atherosclerosis (20).

Although the relationship between GGT and atherosclerosis is well known, the exact mechanisms underlying the relation between GGT and atherosclerosis remain unclear. Possible causes could include some theories. Firstly, GGT is related to many atherosclerotic risk factors. Previous studies showed that serum GGT levels were found to be related to hypertension, metabolic syndrome and DM (1-4). In our study, there was no patient with hypertension, metabolic syndrome or DM. But despite this, in our study GGT levels were found to be associated with VBI and atherosclerotic risk factors such as total cholesterol, LDL cholesterol, HDL cholesterol and triglyceride. This result may indicate that GGT is associated with atherosclerosis alone. The other possible cause may be the relationship between GGT and oxidative stres. GGT is a catalytic enzyme in the extracellular degradation of glutathione which is the major thiol antioxidant in the body. GGT has been shown to be present in the atherosclerotic plaque, acting as a pro-oxidant factor by increasing production of free radicals and reactive oxygen species $(21,22)$. Previous studies showed that an increase in serum GGT concentrations can be used as a marker for increased oxidative stress (23). In addition, serum GGT activities are negatively related with several antioxidants such as beta carotene and vitamin C, and positively associated with oxidative stress parameters such as F-2 isoprostanes $(2,24)$. Another possible cause may be the relationship between inflammation, CRP and GGT. Atherosclerosis is generally accepted as a chronic inflammatory disorder (25). The relationship between GGT and inflammation is well known (26). In the present study, GGT levels were associated with CRP levels. In paralel with our study, previous studies have found significant correlations between GGT and CRP, suggesting that this enzyme represents the expression of subclinical inflammation $(2,26,27)$. In addition to oxidative stres and atherosclerotic processes, systemic inflammation may be a bridge for the relationship between VBI and GGT activity.

In coclusion, although the relationship between GGT, stroke, coronary artery and peripheral arterial disease were evaluated before, the relation between GGT and VBI has not been studied so far. We demonstrated a significant correlation between serum GGT levels and VBI. In addition GGT was an independent risk factor for the presence of vertebrobasilar system atherosclerosis and inflammation. By examining GGT levels, early treatment can be started in patients who were admitted with findings suggest- 
ing VBI such as vertigo, dizziness,etc, and it may be possible to improve the quality of life of these patients.

\section{References}

1. Emdin M, Passino C, Michelassi C, Donato L, Pompella A, Paolicchi A. Additive prognostic value of gamma-glutamyltransferase in coronary artery disease. Int J Cardiol 2009; 136: 80-85.

2. Lee DH, Jacobs DR Jr, Gross M et al. Gamma-glutamyltransferase is a predictor of incident diabetes and hypertension: the Coronary Artery Risk Development in Young Adults (CARDIA) Study. Clin Chem 2003; 49: 1358-1366.

3. Perry IJ, Wannamethee SG, Shaper AG. Prospective study of serum gammaglutamyltransferase and risk of NIDDM. Diabetes Care 1998; 21: 732-737.

4. Bozbaș H, Yıldırır A, Karaçağlar E et al. Increased serum gammaglutamyltransferase activity in patients with metabolic syndrome. Turk Kardiyol Dern Ars 2011; 39: 122-128.

5. Yamasoba T, Kikuchi S, Higo R. Deafness associated with vertebrobasilar insufficiency. J Neurol Sci 2001; 187 (1-2): 69-75.

6. Kwon SU, Kim BJ, Kim SR et al. The response of carotid intimamedia thickness to medical treatment is correlated with that of intracranial atherosclerosis. J Clin Neurol 2013; 9 (4): 231-236.

7. Castaigne P, Lhermitte F, Gautier JC et al. Arterial occlusions in the vertebro-basilar system. A study of 44 patients with post-mortem data. Brain 1973; 96 (1): 133-154.

8. Cloud GC, Markus HS. Diagnosis and management of vertebral artery stenosis. QJM 2003; 96 (1): 27-54.

9. Kuhl V, Tettenborn B, Eicke BM, Visbeck A, Meckes S. Color-coded duplex ultrasonography of the origin of the vertebral artery: normal values of flow velocities. J Neuroimaging 2000; 10 (1): 17-21.

10. Turgut O, Tandogan I. Gamma-glutamyltransferase to determine cardiovascular risk: shifting the paradigm forward. J Atheroscler Thromb 2011; 18: 177-181.

11. Paolicchi A, Emdin M, Ghliozeni $\mathbf{E}$ et al. Images in cardiovascular medicine. Atherosclerotic plaques contain gammaglutamyltranspeptidase activity. Circulation 2004; 109: 1140.

12. Seidel E, Eicke BM, Tettenborn B, Krummenauer F. Reference values for vertebral artery flow volume by duplex sonography in young and elderly adults. Stroke 1999; 30 (12): 2692-2696.

13. Bendick PJ, Glover JL. Vertebrobasilar insufficiency: evaluation by quantitative duplex flow measurements. A preliminary report. J Vasc Surg 1987; 5 (4): 594-600.
14. Boone DJ, Tietz NW, Weinstock A. Significance of gamma-glutamyl transferase (GGT) activity measurements in alcohol-induced hepatic injury. Ann Clin Lab Sci 1977; 7: 25-28.

15. Waters D, Craven TE, Lesperance J. Prognostic significance of progression of coronary atherosclerosis. Circulation 1993; 87: 1067-1075.

16. Aksakal E, Tanboga IH, Kurt M et al. The relation of serum gammaglutamyl transferase levels with coronary lesion complexity and long-term outcome in patients with stable coronary artery disease. Atherosclerosis 2012; 221: 596-601.

17. Higashiyama A, Wakabayashi I, Ono Y et al. Association with serum gamma-glutamyltransferase levels and alcohol consumption on stroke and coronary artery disease: the Suita study. Stroke 2011; 42: 1764-1767.

18. Dogan A, Icli A, Aksoy F et al. Gamma-glutamyltransferase in acute coronary syndrome patients without ST elevation and its association with stenotic lesion and cardiac events. Coron Artery Dis 2012; 23: 39-44.

19. Shankar A, Li J, Klein BE, Nieto FJ, Klein R. Serum gammaglutamyltransferase level and peripheral arterial disease. Atherosclerosis 2008; 199: 102-109.

20. Eroglu S, Sade LE, Polat E, Bozbas H, Ulus T, Muderrisoglu H. Association between serum gamma-glutamyltransferase activity and carotid intima-media thickness. Angiology 2011; 62: 107-110.

21. Pompella A, Emdin M, Passino C, Paolicchi AT. The significance of serum gamma-glutamyltransferase in cardiovascular diseases. Clin Chem Lab Med 2004; 42: 1085-1091.

22. Franzini M, Corti A, Martinelli B et al. Gamma-glutamyltransferase activity in human atherosclerotic plaques - biochemical similarities with the circulating enzyme. Atherosclerosis 2009; 202: 119-127.

23. Emdin M, Pompella A, Paolicchi A. Gamma-glutamyltransferase, atherosclerosis, and cardiovascular disease: triggering oxidative stress within the plaque. Circulation 2005; 112: 2078-2080.

24. Lim JS, Yang JH, Chun BY, Kam S, Jacobs DR Jr, Lee DH. Is serum gamma-glutamyltransferase inversely associated with serum antioxidants as a marker of oxidative stress? Free Radic Biol Med 2004; 37: 1018-1023.

25. Ross R. Atherosclerosis: an inflammatory disease. N Engl J Med 1999; 340: 115-126.

26. Lee DH, Jacobs DR Jr. Association between serum gammaglutamyltransferase and C-reactive protein. Atherosclerosis 2005; 178: 327-330.

27. Demircan S, Yazici M, Durna K et al. The importance of gammaglutamyltransferase activity in patients with coronary artery disease. Clin Cardiol 2009; 32: 220-225.

Received March 7, 2016. Accepted March 24, 2016. 